Rev. Int. Contam. Ambie. 35 (2) 397-406, 2019

DOI: 10.20937/RICA.2019.35.02.11

\title{
PLAGUICIDAS ORGANOCLORADOS EN AGUA DE LA LAGUNA NEGRA DE PUERTO MARQUÉS, ACAPULCO, GUERRERO, MÉXICO
}

\author{
(Organochlorine pesticides in water of the Laguna Negra of Puerto Marques, Acapulco, Guerrero, Mexico)
}

\begin{abstract}
Jesús Clemente SIERRA-CORTÉS, Salvador VEGA Y LEÓN, Rey GUTIÉRREZ-TOLENTINO*, Rutilio ORTIS-SALINAS, José Jesús PÉREZ-GONZÁLEZ y Arturo Camilo ESCOBAR-MEDINA
\end{abstract}

Universidad Autónoma Metropolitana-Unidad Xochimilco, Calzada del Hueso 1100, Col. Villa Quietud, 04960 Ciudad de México, México

*Autor para correspondencia: reygut@correo.xoc.uam.mx

(Recibido diciembre 2017, aceptado julio 2018)

Palabras clave: contaminantes orgánicos, ecosistema costero, Pacífico

\section{RESUMEN}

La presencia de plaguicidas organoclorados en los cuerpos acuáticos es un tema de vital importancia, debido a que se afecta un recurso natural no renovable y con ello la calidad del agua para los organismos vivos que consumen este recurso. Las lagunas costeras son cuerpos acuáticos que reciben una gran cantidad de aportes de materia orgánica y de contaminantes orgánicos (hidrocarburos de petróleo y plaguicidas) e inorgánicos (metales pesados), y donde se efectúan los ciclos biogeoquímicos y biológicos de muchas especies de interés comercial, así como actividades turísticas. El objetivo del presente trabajo fue cuantificar las concentraciones de plaguicidas organoclorados en el agua de la Laguna Negra de Puerto Marqués, Acapulco. Los compuestos organoclorados se determinaron mediante análisis de cromatografía de gases con detector de captura de electrones $\left({ }^{63} \mathrm{Ni}\right)$. Los resultados obtenidos en los sitios de muestreo fueron divididos en cuerpo lagunar y de descarga; no presentaron una diferencia significativa, ya que las concentraciones promedio totales fueron de 5.55 y $6.02 \mu \mathrm{g} / \mathrm{L}$, respectivamente. Asimismo, se apreció que durante la época de estiaje se registró una media total de $6.80 \mu \mathrm{g} / \mathrm{L}$, mientras que en la época de lluvia fue de $3.56 \mu \mathrm{g} / \mathrm{L}$. De acuerdo con la norma oficial mexicana NOM-SSA1-1994 no deben de existir plaguicidas organoclorados, ya que si bien las concentraciones son bajas $(\mu \mathrm{g} / \mathrm{L})$, pueden causar alguna alteración a los organismos por bioacumulación y biomagnificación, con riesgos para la salud del sistema natural y del ser humano.

Key words: organic pollutants, coastal ecosystem, Pacific

\begin{abstract}
The presence of organochlorine pesticides in aquatic systems is an issue of vital importance, because it affects non-renewable natural resources and as consequence the quality of water for organisms that live and use this resource. Coastal lagoons are aquatic systems that receive a large amount of organic matter and organic (petroleum hydrocarbons and pesticides) and inorganic (heavy metals) pollutants, where the
\end{abstract}


biogeochemical and biological cycles of many species of commercial interest are carried out, as well as tourism activities. The objective of the present work was to quantify the concentrations of organochlorine pesticides in the water of the Laguna Negra of Puerto Marqués, Acapulco. The organochlorine compounds were determined by gas chromatography analysis with electron capture detector $\left({ }^{63} \mathrm{Ni}\right)$. The results obtained in the sampling sites were divided into lagoon and discharge sites and did not show a significant difference, since the total average concentrations were 5.55 and $6.02 \mu \mathrm{g} / \mathrm{L}$, respectively. It was also noted that during the dry season a total average of $6.80 \mu \mathrm{g} / \mathrm{L}$ was recorded, while in the rainy season it was $3.56 \mu \mathrm{g} / \mathrm{L}$. According to the Official Mexican Standard NOM-SSA1-1994 there should be no organochlorine pesticides. Although concentrations are low $(\mu \mathrm{g} / \mathrm{L})$, they can cause some alteration to organisms through bioaccumulation and biomagnification with risks to the health of the natural system and the human being.

\section{INTRODUCCIÓN}

En los últimos años se ha observado un deterioro en la calidad del agua de los ecosistemas acuáticos debido al exceso de materia orgánica y fertilizantes químicos, así como la presencia de contaminantes inorgánicos (metales pesados) y orgánicos (hidrocarburos de petróleo, plaguicidas, y antibióticos). La presencia de plaguicidas en el ambiente se debe a su uso excesivo para el control de plagas como hongos, roedores e insectos, entre otros (Matthews 2015), así como en campañas de control de vectores que transmiten enfermedades al ser humano como paludismo, dengue, enfermedad de Chagas, fiebre amarilla y tifoidea, entre otros (Martínez et al. 2011, Mena-Espino y Couoh-Ubicab 2015). Dichas acciones tienden a contaminar los ecosistemas acuáticos y, con ello, alterar el equilibrio de la flora y fauna de los ecosistemas, con graves consecuencias para el reciclaje de nutrientes y la actividad biológica, entre otros. Tal problemática de los plaguicidas tiene su origen en sus propiedades fisicoquímicas, que les confieren una alta persistencia en el ambiente asociada con condiciones climatológicas como humedad, radiación, temperatura, viento y presencia de partículas absorbentes (arcillas, materia orgánica), entre otras (Ohura et al. 2013).

Se ha demostrado la existencia de una relación intrínseca entre los ecosistemas acuáticos costeros y terrestres que alimentan a las lagunas costeras, derivada del aporte de materia orgánica y mineral a través de la erosión hídrica (provenientes de los ríos) y de las actividades humanas (drenajes municipales). De este modo existe una entrada continua de materiales que pueden favorecer o alterar un ecosistema costero (Benavides et al. 2007).
Los compuestos clorados son adsorbidos por las partículas en suspensión (materia orgánica y/o mineral) y, de acuerdo con su tamaño, éstas pueden permanecer en la columna de agua o bien depositarse y acumularse en los sedimentos (Singh et al. 2005a). Una vez depositadas en los sedimentos, éstos pueden regresar al cuerpo de agua mediante resuspensión originada por la actividad del viento o por corrientes subacuáticas (Daskalakis y O'Connor 1995). A través del tiempo y mediante la presencia de microorganismos, los compuestos clorados pueden degradarse en metabolitos secundarios, que suelen ser más tóxicos que los compuestos originales. Esta combinación puede magnificar el efecto tóxico de un contaminante orgánico a bajas concentraciones sobre los seres vivos (Yang et al. 2013). Cuando los compuestos clorados son consumidos por los organismos productores o consumidores primarios, éstos ingresan automáticamente a la red trófica acuática (Lee et al. 2001, Leyva-Cardoso et al. 2003, Singh et al. 2005a).

En México se ha prohibido el uso de algunos plaguicidas organoclorados (POC) como aldrín, endrín y dieldrín (Montelongo 2010, Montes et al. 2012), y otros se siguen empleando de manera restringida en las actividades agrícolas, como el endosulfán. El uso del diclorodifeniltricloroetano (DDT) está prohibido desde 1999 y posteriormente se prohibió el uso del lindano. Con la finalidad de seguir cumpliendo con las obligaciones de país establecidas por los convenios internacionales, y para garantizar que la población no se exponga a plaguicidas considerablemente peligrosos, a partir de 2015 se han cancelado 80 registros sanitarios de ingredientes activos de este tipo de productos (CICOPLAFEST 2019).

Por lo anterior, el objetivo de este estudio fue identificar y cuantificar las concentraciones de los 


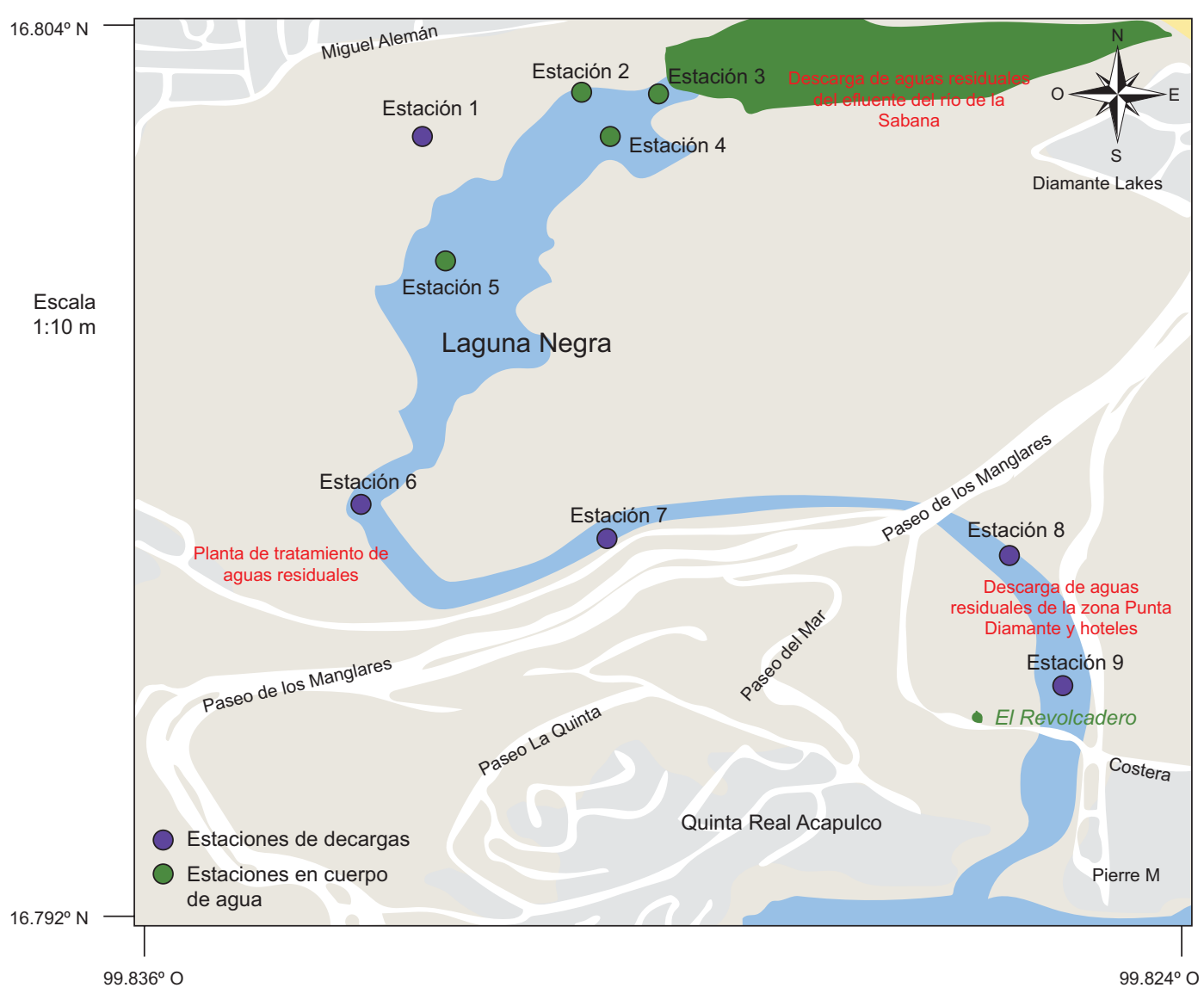

Fig. 1. Fuentes de contaminación por plaguicidas organoclorados y puntos de muestreo en la zona de estudio de la Laguna Negra de Puerto Marqués, Acapulco, Guerrero, México

plaguicidas organoclorados en el agua de la Laguna Negra de Puerto Marqués, Acapulco de Juárez, Guerrero, México, cuya ubicación se muestra en la figura 1, ya que no existen reportes de contaminación por compuestos organoclorados en esta zona.

\section{MATERIALES Y MÉTODOS}

\section{Área de estudio}

La Laguna Negra de Puerto Marqués tiene una superficie de 66.4 ha, está cubierta por mangle casi en su totalidad y tiene una profundidad media de 3.7 $\mathrm{m}$. Forma parte de un sistema hidrológico constituido, además, por el río de la Sabana y la Laguna de Tres Palos. Está localizada en el municipio de Acapulco de Juárez en el estado de Guerrero, México. Se ubica entre los meridianos $16^{\circ} 47^{\prime} 16^{\prime \prime}$ y $17^{\circ} 10^{\prime} 24^{\prime}$ " de latitud norte, y los paralelos $99^{\circ} 39^{\prime} 05^{\prime \prime}$ y $99^{\circ} 53^{\prime} 48^{\prime \prime}$ de latitud oeste (INEGI 2006).

\section{Muestreo y análisis de plaguicidas en agua}

Se eligieron nueve puntos de muestreo a lo largo del canal principal de la Laguna Negra de Puerto Marqués mediante el sistema de posicionamiento global (GPS, por sus siglas en inglés), en los cuales se recolectaron bimestralmente durante un año muestras de agua. La relación de sitios fue la siguiente: cuerpo de la laguna (cuatro puntos) y sitios de descarga (cinco puntos), tomando en cuenta fuentes de contaminación (ríos, drenaje municipal, y la planta tratadora de aguas residuales) (Fig. 1).

Un total de 54 muestras de agua fueron recolectadas de junio de 2012 a abril de 2013. Las muestras se tomaron a una profundidad de $50 \mathrm{~cm}$, en botellas de $4 \mathrm{~L}$ de color ámbar, previamente tratadas con solventes. A las muestras recolectadas in situ se les realizó la extracción con $60 \mathrm{~mL}$ de hexano grado cromatográfico de alta resolución (HPLC) y este volumen se volvió a adicionar para 
obtener un extracto de solvente de $120 \mathrm{~mL}$. E1 extracto orgánico se recogió en frascos de boca ancha de color ámbar y se mantuvo en refrigeración a $4{ }^{\circ} \mathrm{C}$ hasta su análisis. De acuerdo con el método de la Agencia de Protección Ambiental de Estados Unidos (US-EPA 1981), se realizó la extracción y purificación de los analitos que se describe a continuación.

\section{Análisis de plaguicidas}

La purificación de los extractos orgánicos se efectuó a través de columnas cromatográficas empacadas con $13 \mathrm{~g}$ de florisil desactivado $\left(\mathrm{a} 700^{\circ} \mathrm{C}\right.$ por $12 \mathrm{~h}$ y a $400{ }^{\circ} \mathrm{C}$ por $5 \mathrm{~h}$, desactivado con agua tridestilada al $5 \%$ ) únicamente para su uso inmediato y sulfato de sodio anhidro (a $400{ }^{\circ} \mathrm{C}$ por $4 \mathrm{~h}$ ). $\mathrm{La}$ columna se eluyó con 50 y $20 \mathrm{~mL}$ de una solución hexano:éter etílico $(9: 1$ y $8: 2 \mathrm{v} / \mathrm{v})$ para los plaguicidas organoclorados con goteo lento (1 gota/s). Este procedimiento se realizó en un tiempo no mayor a 15 días después de su extracción en campo. Los extractos purificados se concentraron en un sistema de rotaevaporación hasta obtener un volumen de 1 $\mathrm{mL}$; posteriormente se trasvasaron en viales con un flujo de nitrógeno y luego se reconstituyeron con iso-octano grado HPLC, para su análisis en cromatografía de gases, siguiendo el protocolo propuesto por la US-EPA (1981).

\section{Análisis cromatográficos}

La determinación de plaguicidas se realizó en un cromatógrafo de gases (CG) HP 6890 Series con detector de captura de electrones $\left(\mathrm{ECD}^{63} \mathrm{Ni}\right)$ y una columna capilar HP-5MS entrecruzada $5 \%$, metil siloxano $(30 \mathrm{~m}, 0.25 \mathrm{~mm}$ diámetro interno, $0.25 \mu \mathrm{m}$ grosor capa). La temperatura del horno inició a $90^{\circ} \mathrm{C}$ durante $2 \mathrm{~min}$, seguida de una rampa de $30^{\circ} \mathrm{C} / \mathrm{min}$ hasta llegar a $180^{\circ} \mathrm{C}$ por $0 \mathrm{~min}$, con una rampa de $1{ }^{\circ} \mathrm{C} / \mathrm{min}$ para 1 legar a de $200{ }^{\circ} \mathrm{C}$ por $0 \mathrm{~min}$, y posteriormente una rampa de $10{ }^{\circ} \mathrm{C} / \mathrm{min}$ hasta llegar a una temperatura final de $300{ }^{\circ} \mathrm{C}$ durante 6 min. Se utilizó helio como gas acarreador y nitrógeno como gas auxiliar. Las condiciones de temperatura del CG en el inyector y el detector fueron de 320 ${ }^{\circ} \mathrm{C}$. Las concentraciones finales se determinaron utilizando un estándar externo de 16 compuestos organoclorados (Chem Service, organochlorine pesticides mixture 608/625/8080/8081): 1) alfa$\mathrm{HCH}, 2)$ beta-HCH, 3) gama-HCH, 4) delta- $\mathrm{HCH}$, 5) heptacloro, 6) aldrín, 7) epóxido de heptacloro, 8 ) endosulfán I, 9) p, $p^{\prime}$-DDE, 10) dieldrín, 11) endrín, 12) endosulfán II, 13) p,p'-DDD, 14) endrín aldehído, 15) endosulfato y 16) p,p'-DDT.

\section{Cuantificación y parámetros del desempeño del método}

La identificación de los analitos de plaguicidas organoclorados se realizó mediante una solución estándar de una mezcla de 16 compuestos con el procesador de datos EZ Chrom Elite Versión 3.3.2 SP2, basado en los tiempos de retención y la cuantificación de POC a partir del área debajo del pico (factor de respuesta, $\mathrm{mVs}$ ) vs. la curva de calibración. El límite de detección y cuantificación se estableció a partir de 3 y 10 veces el valor de la desviación estándar de la concentración más baja de la curva de calibración de cada POC (Cuadro I). El recobrado se obtuvo con las concentraciones calculadas para cada POC a partir de muestras adicionadas y en blancos fortificados al inicio de la extracción de analitos, seguida de la purificación en columnas cromatográficas y concentración de los extractos hasta su análisis en cromatografía de gases, las cuales variaron de 76 a $87 \%$, en tanto que los compuestos endosulfán I y II, y endosulfato variaron de 100 a $130 \%$ (Cuadro I).

CUADRO I. VALORES DE LÍMITE DE DETECCIÓN Y DESVIACIÓN ESTÁNDAR) EN EL RECOBRADO PARA PLAGUICIDAS ORGANOCLORADOS

\begin{tabular}{lccc}
\hline Compuestos & $\begin{array}{c}\text { DE } \\
(\%)\end{array}$ & $\begin{array}{c}\text { LD } \\
(\mathrm{ng} / \mathrm{mL})\end{array}$ & $\begin{array}{c}\text { Recobrado } \\
(\%)\end{array}$ \\
\hline alfa-HCH & 6 & 0.08 & 76 \\
beta-HCH & 3 & 0.16 & 87 \\
gama-HCH & 5 & 0.08 & 83 \\
delta-HCH & 4 & 0.08 & 85 \\
Heptacloro & 8 & 0.15 & 75 \\
Aldrín & 7 & 0.08 & 78 \\
Epóxido de heptacloro & 3 & 0.08 & 76 \\
Endosulfán I & 1 & 0.02 & 100 \\
DDE & 7 & 0.08 & 80 \\
Dieldrín & 4 & 0.15 & 78 \\
Endrin & 6 & 0.10 & 87 \\
Endosulfán II & 1 & 0.02 & 105 \\
DDD & 5 & 0.08 & 79 \\
Endrín aldehído & 6 & 0.07 & 85 \\
Endosulfato II & 1 & 0.02 & 130 \\
DDT & 5 & 0.08 & 87 \\
\hline
\end{tabular}

DE: desviación estándar; $\mathrm{HCH}$ : hexaclorociclohexano, DDE: diclorodifenildicloroetileno, DDD: diclorodifenildicloroetano, DDT: diclorodifeniltricloroetano

Con las concentraciones obtenidas se aplicó un análisis de estadística descriptiva con el programa SSPS v. X para Windows y el empleo de diagramas de caja y bigote. 


\section{RESULTADOS Y DISCUSIÓN}

En el cuadro II se muestran los resultados de concentraciones de POC en agua para los sitios de cuerpo de la laguna y de descarga, entre los cuales son muy variables . Por ejemplo, en los sitios de descarga las concentraciones de endosulfán II, dieldrín, beta-HCH, DDT y DDD fueron de 0.68-33.30 $\mu \mathrm{g} / \mathrm{L}$, $0.20-71.70 \mu \mathrm{g} / \mathrm{L}, 0.10-91.50 \mu \mathrm{g} / \mathrm{L}, 0.90-11.30 \mu \mathrm{g} / \mathrm{L}$ y $0.00-4.30 \mu \mathrm{g} / \mathrm{L}$, respectivamente; mientras que, en el caso de sitios designados como cuerpo de agua, las concentraciones de endosulfato II, epóxido de heptacloro, gama-HCH, DDT, alfa-HCH y endosulfán II fueron de 2.10-34.30 $\mu \mathrm{g} / \mathrm{L}, 2.60-17.20 \mu \mathrm{g} / \mathrm{L}$, $0.30-34.40 \mu \mathrm{g} / \mathrm{L}, 0.40-20.20 \mu \mathrm{g} / \mathrm{L} \quad 0.40-27.80 \mu \mathrm{g} / \mathrm{L}$ y $0.10-1.80 \mu \mathrm{g} / \mathrm{L}$ (siendo esta última la más baja), respectivamente. Se aprecia que el endosulfato se presentó con valores altos en ambos sitios de muestreo. Por lo tanto, no existe una diferencia significativa en ambos sitios.

Los sitios de descarga y cuerpos de la Laguna exhiben un mismo grado de afectación determinado por el ingreso de descargas del río hacia la laguna, donde la corriente de agua influye en la distribución de los compuestos para que se acumulen en sitios centrales del cuerpo acuático. En éste destaca la mayor presencia de los isómeros del HCH (beta y gama). De acuerdo con lo reportado por Georgina y Torres (1998), una vez que ingresan a los ecosistemas acuáticos, los plaguicidas se mueven por advección (movimiento que depende de la velocidad y dirección de las corrientes de agua).

\section{Comportamiento de los plaguicidas en época de estiaje y lluvia}

En el cuadro III se aprecian las distribuciones de los plaguicidas organoclorados en función de las temporadas de lluvia (junio-octubre) y estiaje (noviembre-abril) durante el periodo 2012-2013. En general, las mayores concentraciones se presentaron en la época de estiaje, en la cual hay mayor evaporación y por tanto aumenta la concentración de contaminantes en el agua, situación que no se apreció con el DDT. La concentración total promedio de los plaguicidas en época de estiaje fue de $6.80 \mu \mathrm{g} / \mathrm{L}$ mientras que en la época de lluvia fue de $3.57 \mu \mathrm{g} / \mathrm{L}$. Para la época seca, los compuestos más concentrados fueron en promedio los siguientes: endosulfato $(23.15 \mu \mathrm{g} / \mathrm{L})$, alfa-HCH $(8.34 \mu \mathrm{g} / \mathrm{L})$, gama-HCH $(7.98 \mu \mathrm{g} / \mathrm{L})$, dieldrín $(7.25 \mu \mathrm{g} / \mathrm{L})$, beta-HCH $(7.23$ $\mu \mathrm{g} / \mathrm{L})$ y el compuesto con menor concentración fue el endosulfán II $(3.51 \mu \mathrm{g} / \mathrm{L})$.

En la temporada de lluvia, los compuestos con mayor concentración promedio fueron DDT

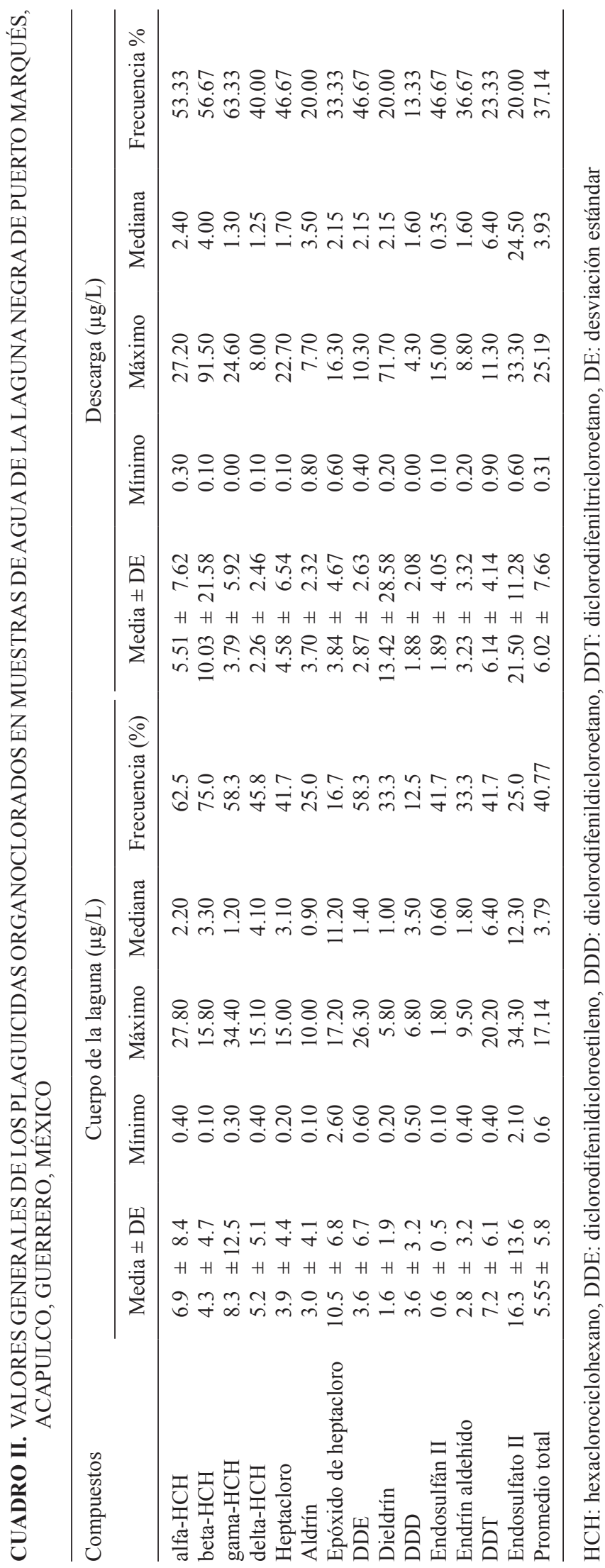


CUADRO III. VALORES DE PLAGUICIDAS ORGANOCLORADOS EN MUESTRAS DE AGUA DE LA LAGUNA NEGRA DE PUERTO MARQUÉS, ACAPULCO, GUERRERO, MÉXICO, EN ÉPOCAS DE LLUVIAS Y ESTIAJE

\begin{tabular}{|c|c|c|c|c|c|c|}
\hline \multirow{3}{*}{ Compuestos } & \multicolumn{6}{|c|}{ Concentraciones $(\mu \mathrm{g} / \mathrm{L})$} \\
\hline & \multicolumn{3}{|c|}{ Época de lluvia } & \multicolumn{3}{|c|}{ Época de estiaje } \\
\hline & Media \pm DE & Mínimo & Máximo & Media $\pm \mathrm{DE}$ & Mínimo & Máximo \\
\hline alfa-HCH & $2.2 \pm 2.5$ & 0.40 & 9.3 & $8.34 \pm 8.90$ & 0.30 & 27.80 \\
\hline beta-HCH & $6.8 \pm 6.8$ & 0.20 & 20.6 & $7.23 \pm 18.97$ & 0.10 & 91.50 \\
\hline gama-HCH & $1.7 \pm 3.5$ & 0.30 & 12.80 & $7.98 \pm 10.93$ & 0.00 & 34.40 \\
\hline delta-HCH & $3.0 \pm 5.9$ & 0.10 & 15.10 & $3.91 \pm 3.50$ & 0.10 & 15.10 \\
\hline Heptacloro & $1.1 \pm 1.3$ & 0.20 & 3.80 & $5.60 \pm 6.22$ & 0.10 & 22.70 \\
\hline Aldrín & $0.2 \pm 0.0$ & 0.20 & 0.20 & $3.63 \pm 3.17$ & 0.10 & 10.00 \\
\hline Epóxido de heptacloro & $5.1 \pm 0.0$ & 5.1 & 5.10 & $5.80 \pm 6.21$ & 0.60 & 17.20 \\
\hline DDE & $1.6 \pm 0.6$ & 0.60 & 2.70 & $4.48 \pm 6.40$ & 0.40 & 26.30 \\
\hline Dieldrín & $3.1 \pm 1.1$ & 2.30 & 3.80 & $7.25 \pm 20.36$ & 0.20 & 71.70 \\
\hline DDD & $0.1 \pm 0.2$ & 0.00 & 6.30 & $3.60 \pm 2.28$ & 0.50 & 6.80 \\
\hline Endosulfán II & $0.4 \pm 0.3$ & 0.10 & 1.10 & $3.51 \pm 5.43$ & 0.10 & 15.00 \\
\hline Endrín aldehído & $1.42 \pm 2.1$ & 0.20 & 7.20 & $4.89 \pm 3.31$ & 0.40 & 9.50 \\
\hline DDT & $12.8 \pm 10.4$ & 5.50 & 20.20 & $5.95 \pm 4.27$ & 0.40 & 13.20 \\
\hline Endosulfato II & $10.4 \pm 15.4$ & 0.60 & 33.30 & $23.15 \pm 8.45$ & 8.30 & 34.30 \\
\hline Promedio total & $3.56 \pm 13.7$ & 0.0 & 47.40 & $6.80 \pm 37.66$ & 0.00 & 150.00 \\
\hline
\end{tabular}

$(12.85 \mu \mathrm{g} / \mathrm{L})$, endosulfato II $(10.45 \mu \mathrm{g} / \mathrm{L})$ y beta$\mathrm{HCH}(6.79 \mu \mathrm{g} / \mathrm{L})$, y el que presentó menores valores fue el DDD $(0.15 \mu \mathrm{g} / \mathrm{L})$. Al existir un mayor volumen de agua en la laguna, se aprecia un efecto de dilución, pero a la vez existe un ingreso de contaminantes, dado que el río Sabana alimenta al cuerpo acuático, y dicha vía fluvial transporta las descargas de varios municipios agrícolas ubicados en la parte superior de la cuenca hidrológica y de los asentamientos urbanos cercanos, como Ciudad Renacimiento, el rastro municipal de la Sabana, y la planta de tratamiento de aguas residuales del poblado de Puerto Marqués y de la zona hotelera cercana a la laguna (Hotel Princess).

Los suelos de zonas agrícolas son reservorios de productos químicos como los POC, como resultado del efecto de lixiviación y depósito por vía atmosférica (Schuster et al. 2011). Los plaguicidas en el suelo sufren degradación química o transformación metabólica por bacterias por diferentes condiciones (Chandra et al. 2016). En el caso del endosulfán técnico (una mezcla de dos isómeros alfa y beta-endosulfán en proporciones aproximadas de 70 y $30 \%$, respectivamente), éste puede transformarse en endosulfato por la acción de los microbios del suelo. Por lo tanto, debido a las precipitaciones pluviales, estos compuestos clorados se incorporan a los cuerpos acuáticos por acción de la escorrentía y lixiviación de elementos inorgánicos y orgánicos (Cotham y Bidelman 1989, Gil et al. 2012).

La aparición del endosulfán I y II sugiere una degradación del compuesto principal endosulfán, lo que puede indicar una deposición histórica dada la existencia de compuestos derivados de la degradación, en función de las condiciones de microorganismos aerobios en la parte superficial de la laguna. La presencia de compuestos de degradación en lagunas costeras se han reportado como tóxica para la vida acuática (Naqvi y Vaishnavi 1993, Botello et al. 2000, Tripathi y Verma 2004, Carvalho et al. 2009).

En función de la composición química de los plaguicidas organoclorados, éstos se clasifican en alicíclicos (alfa-, beta-, gama- y delta-HCH), ciclodienos (heptacloro, epóxido de heptacloro, dieldrín, endrín, endosulfán I y II, endosulfato, endrín aldehído y aldrín) y aromáticos (DDT, DDE y DDD). En las muestras estudiadas de la laguna se detectaron estos tres grupos de plaguicidas organoclorados. El grupo de los alicíclicos se presentó en mayor porcentaje en los sitios de muestreo, en un rango de 45.8 y $75 \%$. De acuerdo con Chandra et al. (2016), la aparición del gama-HCH se debe a su uso en el control de plagas agrícolas y en el tratamiento de la sarna (NIPN 2007), al igual que la del beta-HCH, un isómero más persistente del lindano. 
Desde la década de 1980 se prohibió el uso de algunos plaguicidas organoclorados, como fue el caso del HCH técnico (denominado BHC) y del DDT, debido a sus efectos negativos en el ambiente y su baja eficacia contra las plagas. Cabe mencionar que si bien el DDT ha sido prohibido para fines agrícolas en México y se signó el convenio de Estocolmo para la restricción de dicho plaguicida, en lugares tropicales se sigue utilizando en campañas de salud pública para el combate de vectores como los mosquitos trasmisores del paludismo (Pérez et al. 2013). Por ello, aunque dicho compuesto se dejó de emplear desde 2000, sigue detectándose en muestras ambientales y en tejido humano (Yáñez et al. 2002, Pérez-Maldonado et al. 2006).

Para entender la presencia de algunos plaguicidas y sus metabolitos en el ambiente es necesario considerar algunas situaciones que se han descrito en diversos trabajos del área ambiental. Por ejemplo, el $\mathrm{HCH}$ de grado técnico está constituido de la siguiente manera: alfa- $\mathrm{HCH}, 60-70 \%$; beta- $\mathrm{HCH}$, 5-14\%; gama-HCH, 8-15\%; delta-HCH, 2-16\%, y, finalmente, el resto de los isómeros, 1-5\%) (Willett et al. 1998, Breivik et al. 1999). La presencia del isómero con mayor porcentaje fue el beta-HCH con $50 \%$ en la época de lluvia, en comparación con la época de estiaje, cuando el isómero alfa- $\mathrm{HCH}$ tuvo el mayor porcentaje con $30 \%$. Esto sugiere aplicaciones recientes de lindano (gama-HCH), de acuerdo con Da et al. (2014), quienes establecen que valores $<3$ son indicativos de lindano, mientras que valores de 3 a 7 corresponden al grado técnico, por lo que existe una degradación del compuesto puro probablemente en un ambiente anaeróbico. Asimismo, el resultado de la relación del alfa $\mathrm{HCH} /$ gama $\mathrm{HCH}$, demuestra el uso del $\mathrm{HCH}$ grado técnico, cuya mezcla contiene varios isómeros de $\mathrm{HCH}$, en general alfa-HCH y beta-HCH (Li et al. 1998, 2001, van de Zelm et al. 2009; Fig. 2).

En general, las concentraciones totales de plaguicidas fueron mayores en la época de estiaje, cuando se observó la contribución de algunos sitios de descarga, en este caso algunos riachuelos que cruzan la zona habitacional cercana a la Laguna donde constantemente se emplean plaguicidas.

En la época de lluvias se incrementó el nivel de agua de la laguna y con ello, se presentó el fenómeno de dilución. Además, debe considerarse la interacción con el mar por medio de las mareas y los vientos que afectan a la Laguna Negra. Por lo tanto, dicho sistema lagunar tiene un intercambio continuo con el suelo (escorrentía y lixiviación) y con la atmósfera (precipitación húmeda y seca) (Pérez-Ruzafa et al. 2000), así como un intercambio de masas de agua a través de las corrientes.

En el caso de los plaguicidas aromáticos, se aplicó un cociente descrito en investigaciones de contaminación de sistemas acuáticos, para conocer si la aplicación del DDT es reciente o histórica. El compuesto principal DDT fue metabolizado a su metabolito diclorodifenildicloroetileno (DDE) en condiciones aeróbicas (Singh et al. 2005b); en cuanto al diclorodifenildicloroetano (DDD), esto ocurrió en ambientes anaeróbicos (Yang et al. 2013). Una proporción de (DDE + DDD)/DDT $>1$ sugiere contaminación histórica, en tanto que

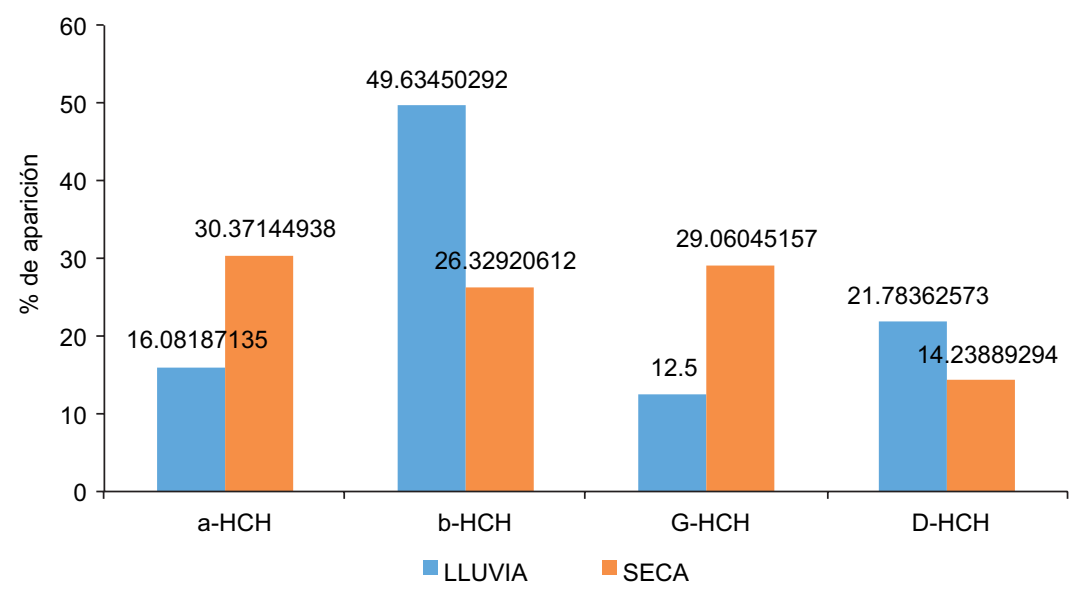

Fig. 2. Porcentaje de aparición y coeficiente de relación alfa-HCH/gama-HCH (hexaclorociclohexano) en muestras de agua superficial de la Laguna Negra de Puerto Marqués 
valores $<1$ se consideran como el resultado de una entrada o fuente reciente de DDT. Los valores de (DDE + DDD)/DDT reportados durante las épocas de lluvias y seca fueron de 0.13 y $1.36 \mu \mathrm{g} / \mathrm{L}$, respectivamente, y se pudo apreciar que durante la época de lluvias dicha proporción fue $<$ a 1 , lo cual puede deberse al deslave del DDT acumulado en el suelo superficial. Se sabe que la Secretaría de Salud en México es la única institución que emplea y aplica DDT en las zonas costeras, de ahí el arrastre de partículas o la volatilización del plaguicida (Pérez-Maldonado et al. 2006). OchoaRivero et al. (2017) afirman que el DDT aún se emplea en México en la agricultura debido a su efectividad y asequibilidad, como demuestran los estudios de análisis de agua superficial del norte del país; esto, aunado a la falta de un inventario real del uso de plaguicidas en las regiones agrícolas del territorio nacional. En general, la proporción (DDE + DDD)/DDT para la época seca fue $>1$, lo que demuestra acumulación y degradación del DDT (Fig. 3) a través del tiempo, considerando que existe una tendencia a la concentración al disminuir el volumen del agua de la laguna (Jaga y Dharmani 2003).

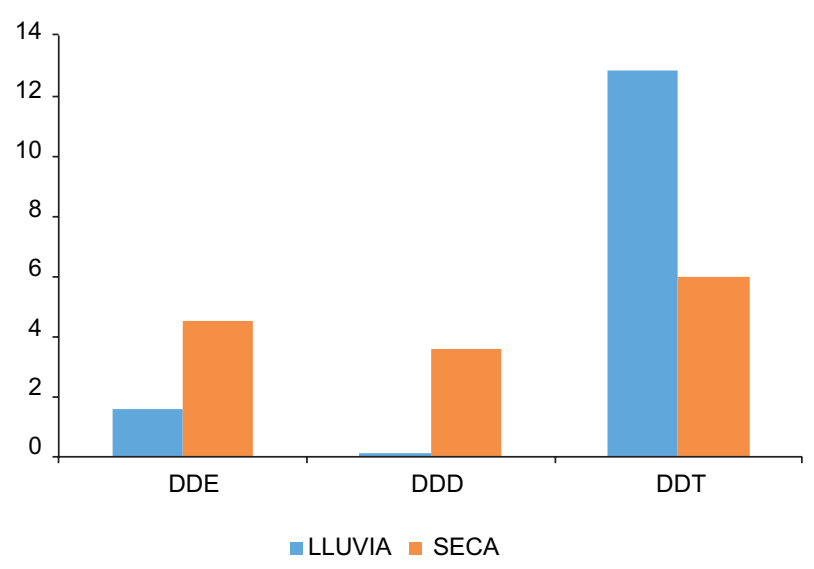

Fig. 3. Concentraciones de diclorodifeniltricloroetano (DDT), diclorodifenildicloroetano (DDD) y diclorodifenildicloroetileno (DDE) en épocas de lluvias y estiaje, así como las proporciones (DDE + DDD)/DDT y DDE/DDD en muestras de agua superficial de la Laguna Negra de Puerto Marqués

En cuanto a la relación DDE/DDD, se apreció una mayor presencia de DDE en ambas épocas (de lluvias y seca) con relación al DDD; dicho comportamiento se puede explicar en razón de que el DDD se degrada más rápido en el ambiente y existe una entrada reciente debido a la prohibición del uso de DDT (Youssef et al. 2015). De acuerdo con Steyn et al. (2018), el DDT se ha empleado para el control del paludismo en Sudáfrica, aspecto que ha sido objeto de reflexión en convenios internacionales, donde se menciona su uso continuado por algunos países (principalmente en vías de desarrollo) para el control de esta enfermedad, a pesar de su prohibición (SCPOP 2008). Asimismo, Polanco et al. (2018) destacan que a pesar de la prohibición de los plaguicidas organoclorados, éstos aún se emplean en países en vías de desarrollo para actividades agrícolas, ganaderas y para el control de vectores en salud pública con el fin de prevenir enfermedades trasmisibles como el dengue y el paludismo.

\section{CONCLUSIONES}

A partir de los resultados de este estudio puede apreciarse que se detectaron 14 de los 16 compuestos organoclorados denominados como prioritarios, con concentraciones referidas en $\mu \mathrm{g} / \mathrm{L}$. El aporte de plaguicidas al sistema lagunar ocurre través de los riachuelos que cruzan las zonas habitacionales cercanas a la Laguna, y se estima un efecto estacional de mayor concentración de plaguicidas en la época de estiaje.

Los compuestos organoclorados dominantes en la Laguna fueron los isómeros de $\mathrm{HCH}$, así como de DDT, DDD y DDE. La presencia de los compuestos mencionados es resultado de su persistencia en el ambiente, ya que a pesar de su prohibición o restricción se siguen detectando en el sistema lagunar. Es importante estudiar el comportamiento de dichos contaminantes en este sistema lagunar costero, ya que se aprecia una alteración en la calidad del agua y con ello una probable amenaza a la diversidad de la fauna en dicho sistema.

\section{AGRADECIMIENTOS}

Al Posgrado de Ciencias Agropecuarias de la Universidad Autónoma Metropolitana-Xochimilco por el apoyo otorgado al desarrollo de la maestría de Jesús Clemente Sierra-Cortés. A la Cooperativa de Pescadores de la Laguna Negra de Puerto Marqués por su apoyo en la recolección de muestras de agua, sedimento y peces. Al Consejo Nacional de Ciencia y Tecnología (CONACYT) por el apoyo económico otorgado. 


\section{REFERENCIAS}

Benavides A., Moreno M., Sosa M., Puga, S., Alcalá J. y Quintana C. (2007). Evaluación de la calidad del agua en las principales lagunas del estado de Chihuahua. Rev. Latinoam. Rec. Nat. 4 (2), 84 -88.

Botello A.V., Rueda-Quintana L., Díaz-González G. y Toledo A. (2000). Persistent organochlorine pesticides (POPs) in coastal lagoons of the subtropical Mexican Pacific. Bull. Environ. Contam. Toxicol. 64 (3), 390397. DOI: $10.1007 / \mathrm{s} 001280000013$

Breivik K., Pacyna J.M. y Münch J. (1999). Use of alpha-, beta- and gamma-hexachlorocyclohexane in Europe, 1970-1996. Sci. Total Environ. 239 (1), 151-163.

DOI: $10.1016 / \mathrm{S} 0048-9697(99) 00291-0$

Carvalho F.P., Villeneuve J.P., Cattin C., Rendón J. y Mota de Oliveira J. (2009). Pesticide and PCB residues in the aquatic ecosystems of Laguna de Términos, a protected area of the coast of Campeche, Mexico. Chemosphere 74 (7), 988-995.

DOI: 10.1016/j.chemosphere.2008.09.092

Chandra Y.I., Linthoingambi D.N., Li J., Zhang G. y Raj S.O. (2016). Occurrence, profile and spatial distribution of organochlorines pesticides in soil of Nepal: Implication for source apportionment and health risk assessment. Sci. Total Environ. 573 (15), 1598-1606. DOI: $10.1016 /$ j.scitotenv.2016.09.133

CICOPLAFEST (2019). Registro Sanitario de Plaguicidas y Nutrientes Vegetales. Registros sanitarios de plaguicidas cancelados [en línea]. https:/www.gob.mx/ cofepris/acciones-y-programas/registro-sanitario-deplaguicidas-y-nutrientes-vegetales y http://siipris03. cofepris.gob.mx/Resoluciones/Consultas/ConWebRegPlaguicidaCancel.asp. 26/04/2019

Cotham W.E. Jr. y Bidleman T.F. (1989). Degradation of malathion, endosulfan and fenvalerate in seawater and seawater/sediment microcosms. J. Agric. Food Chem. 37 (3), 824-828. DOI: 10.1021/jf00087a055

Da C., Liu G. y Yuan Z. (2014). Analysis of HCHs and DDTs in a sediment core from the Old Yellow River Estuary, China. Ecotoxicol. Environ. Saf. 100, 171177. DOI: $10.1016 /$ j.ecoenv.2013.10.034

Daskalakis K.D. y O'connor T.P. (1995). Distribution of chemical contamination in US coastal and estuarine sediments. Mar. Environ. Res. 40 (4), 381-398. DOI: 10.1016/0141-1136(94)00150-N

Georgina C.L. y Torres M.R. (1998). Plaguicidas organoclorados. Contactos 30, 35-46.

Gil M.J., Soto A.M., Usma J.I. y Gutiérrez O.D. (2012). Contaminantes emergentes en aguas, efectos y posibles tratamientos. Producción + Limpia 7 (2), 52-73.

INEGI (2006). Acapulco de Juárez, estado de Guerrero. Cuaderno estadístico municipal. Edición 2006 [en línea]. https://www.inegi.org.mx/app/biblioteca/ficha. html?upc $=702825006534$ 26/04/2019

Jaga K. y Dharmani C. (2003). Global surveillance of DDT and DDE levels in human tissues. Int. J. Occup. Med. Environ. Health. 16 (1), 7-20.

DOI: $10.2478 / \mathrm{v} 10001-006-0009-6$

Lee K.T., Tanabe, S. y Koh C.H. (2001). Distribution of organochlorine pesticides in sediments from Kyeonggi Bay and nearby areas, Korea. Environ. Pollut. 114 (2), 207-213. DOI: 10.1016/S0269-7491(00)00217-7

Leyva-Cardoso D.O., Ponce-Vélez G., Botello A.V. y Díaz-González G. (2003). Persistent organochlorine pesticides in coastal sediments from Petacalco Bay, Guerrero, Mexico. Bull. Environ. Contam. Toxicol. 71 (6), 1244-1251. DOI: 10.1007/s00128-003-8866-1

Li Y.F., Cai D.J. y Sing A. (1998). Technical hexachlorocyclohexane use trends in China and their impact on the environment. Arch. Environ. Contam. Toxicol. 35 (4), 688-697. DOI: 10.1007/s002449900432

Li Y.F., Cai D.J., Shan Z.J. y Zhu L.Z. (2001). Gridded usage inventories of technical hexachlorocyclohexane and lindane for China with $1 / 6$ latitude by $1 / 4$ longitude resolution. Arch. Environ. Contam. Toxicol. 41, 261266. DOI: $10.1007 / \mathrm{s} 002440010247$

Martínez-Salinas R.I., Díaz-Barriga F., Batrés-Esquivel L.E. y Pérez-Maldonado I.N. (2011). Assessment of the levels of DDT and its metabolites in soil and dust samples from Chiapas, Mexico. Bull. Environ. Contam. Toxicol. 86 (1), 33-37. DOI: $10.1007 / \mathrm{s} 00128-010-0174-y$

Matthews G. (2015). Pesticides: Health, safety and the environment. 2nd ed. Wiley Blackwell, 296 pp.

Mena-Espino X. y Couoh-Uicab Y. (2015). Efectos de los plaguicidas utilizados para el control de la sigatoka negra en plantaciones bananeras en México, así como su efecto en el ambiente y la salud pública. Tecnociencia Chihuahua 9 (2), 91-98.

Montelongo B.I. (2010). La regulación jurídica de los plaguicidas en México [en línea]. http://www.azc. uam.mx/publicaciones/alegatos/pdfs/28/31-10.pdf $10 / 01 / 2012$

Montes A.M., Gonzáles-Farías F.A. y Botello A.V. (2012). Pollution by organochlorine pesticides in NavachisteMacapule, Sinaloa, Mexico. Environ. Monit. Assess. 184 (3), 1359-1369. DOI: 10.1007/s10661-011-2046-2

Naqvi S.M. y Vaishnavi C. (1993). Bioaccumulative potential and toxicity of endosulfan an insecticide to non-target animals. Mini-review. Com. Biochem. Physiol. Part C. 105 (3), 347-361.

DOI: 10.1016/0742-8413(93)90071-R

NIPN (2007). The national implementation plan for the Stockholm convention on persistent organic pollutants. National Implementation Plan for Nepal. Ministry of 
Environment, Science and Technology, Singh Darbar, Government of Nepal, Kathmandu Nepal [en línea]. https://www.informea.org/sites/default/files/importeddocuments/UNEP-POPS-NIP-Nepal-1.English.pdf 25/10/2017

Ochoa-Rivero J.M., Reyes-Fierro A.V., Peralta-Pérez M.R., Zavala-Díaz S.F.J., Ballinas-Casarrubias. L., Salmerón I., Rubio-Arias H. y Rocha-Gutiérrez B. (2017). Levels and distribution of pollutants in the waters of an aquatic ecosystem in Northern Mexico. Int. J. Environ. Res. Public. Health. 14 (5), 456-468. DOI: $10.3390 /$ ijerph14050456

Ohura T., Horii Y., Kojima M. y Kamiya Y. (2013). Diurnal variability of chlorinated polycyclic aromatic hydrocarbons in urban air. Atmos. Environ. 81, 84-91, DOI: 10.1016/j.atmosenv.2013.08.044

Pérez-Maldonado I.N., Athanasiadou M., Yánez L., González-Amaro R., Bergman A. y Díaz-Barriga F. (2006). DDE-induced apoptosis in children exposed to the DDT metabolite. Sci. Total Environ. 370 (2), 343-351. DOI: 10.1016/j.scitotenv.2006.06.026

Pérez-Ruzafa A., Navarro S., Barba A., Marcos C., Cámara M.A., Salas F. y Gutiérrez J.M. (2000). Presence of pesticides throughout trophic compartments of the food wed in the Mar Menor Lagoons (SE Spin). Mar. Pollut. Bull. 40 (2), 140-151.

DOI: 10.1016/S0025-326X(99)00193-9

Polanco R.A.G., Riba L.M.I., del Valls C.A., Araujo L.J.A. y Datta B.S. (2018) Impact of pesticides in karst groundwater. Review of recent trends in Yucatan, Mexico. Groundwater Sust. Develop. 7, 20-29.

DOI: $10.1016 /$ j.gsd.2018.02.003

Schuster J.K., Gioia R., Moeckel C., Agarwal T., Bucheli T.D., Breivik K., Steinnes E. y Jones K.C. (2011). Has the burden and distribution of PCBs and PBDEs changed in European background soils between 1998 and 2008? implications for sources and processes. Environ. Sci. Technol. 45 (17), 7291-7297.

DOI: $10.1021 / \mathrm{es} 200961 \mathrm{p}$

SCPOP (2008). Stockholm Convention. The 12 initial POPs under the Stockholm Convention [en línea]. http://chm.pops.int/TheConvention/ThePOPs/ The12InitialPOPs/tabid/296/Default.aspx 26/04/2019

Singh K.P., Malik A., Mohan D. y Takroo R. (2005a). Distribution of persistent organochlorine pesticide residues in Gomti River, India. Bull. Environ. Contam. Toxicol. 74 (1), 146-154. DOI: 10.1007/s00128-004-0561-3
Singh K.P., Malik A., Mohan D. y Sinha S. (2005b). Persistent organochlorine pesticide residues in alluvial groundwater aquifers of Gangetic Plains, India. Bull. Environ. Contam. Toxicol. 74 (1), 162-169.

DOI: $10.1007 / \mathrm{s} 00128-004-0563-1$

Steyn L., Bouwman H. y Maina J.N. (2018). Associations between DDT and egg parameters of the house sparrow Passer domesticus from the Thohoyandou area of South Africa. Chemosphere 198, 249-256. DOI: 10.1016/j.chemosphere.2018.01.125

Tripathi G. y Verma P. (2004). Endosulfan-mediated biochemical changes in the freshwater fish Clarias batrachus. Biomed. Environ. Sci. 17 (1), 47-56.

US-EPA (1981). Manual for analytical quality control for pesticides and related compounds in human and enviromental samples. Second revision. EPA-600/2 81059. United States Enviromental Protection Agency, Washington, EUA. 455 pp.

Van de Zelm R., Huijbregts M.A.J., Posthuma L., Wintersen, A. y van de Meent D. (2009). Pesticide ecotoxicological effect factors and their uncertainties for freshwater ecosystems. Int. J. Life Cycle Assess. 14 (1), 43-51. DOI: $10.1007 / \mathrm{s} 11367-008-0037-5$

Willett K.L., Ulrich E.M. y Hites R.A. (1998). Differential toxicity and environmental fates of hexachlorocyclohexane isomers. Environ. Sci. Technol. 32 (15), 21972207. DOI: $10.1021 /$ es 9708530

Yang D., Qi S., Zhang J., Wu C. y Xing X. (2013). Organochlorine pesticides in soil, water and sediment along the Jinjiang river mainstream to Quanzhou bay, southeast China. Ecotoxicol. Environ. Saf. 89, 59-65. DOI: 10.1016/j.ecoenv.2012.11.014

Yánez L., Ortiz-Pérez D., Batres L.E., Borja-Aburto V.H. y Díaz-Barriga F. (2002). Levels of dichlorodiphenyltrichloroethane and deltamethrin in humans and environmental samples in malarious areas of Mexico. Environ. Res. 88 (3), 174-181. DOI: 10.1006/enrs.2002.4333

Youssef L., Younes G., Kouzayha A. y Jaber F. (2015). Occurrence and levels of pesticides in South Lebanon water. Chem. Spec. Bioavailab. 27 (2), 62-70.

DOI: $10.1080 / 09542299.2015 .1023092 z$ 